\title{
The problem of joint scheduling and power assignment in wireless sensor networks
}

\author{
Ada Gogu*, Supriyo Chatterjea ${ }^{\dagger}$, Dritan Nace* Arta Dilo ${ }^{\dagger}$, \\ *Université de Technologie de Compiègne \\ 60205 Compiègne Cedex, France \\ \{ada.gogu,dnace\}@hds.utc.fr \\ †Pervasive Systems group, EWI \\ University of Twente \\ 7500 AE Enschede, The Netherlands \\ \{s.chatterjea,a.dilo\}@utwente.nl
}

\begin{abstract}
In this work we address the problem of joint link scheduling and power assignment in WSNs. It focuses on finding a feasible schedule and a power assignment scheme such that the schedule length is minimized and the concurrent transmissions have a fair quality in terms of SINR (Signal-to-Interferenceand-Noise Ratio). As the problem is shown to be $\mathcal{N} \mathcal{P}$ hard we propose a greedy heuristic for the scheduling problem which seeks to minimize the number of time slots for link scheduling. Our goal is to design an algorithm that performs transmission power control in order to guarantee fair SINR link transmissions. This insures that every node that is actively transmitting in the network chooses a transmission power which will minimally affect the other concurrent transmissions. Hence, we opt for a cooperative strategy which intends to maximize the minimum value of SINR. This problem is modeled and solved using an iterative algorithm based on linear programming which provides an optimal solution. The solution of this problem may be crucial for the performance of WSNs as it impacts different network requirements such as lifetime (energy savings), delay (schedule length) and throughput (number of concurrent links in a time slot).
\end{abstract}

Index Terms - WSN, power assignment, scheduling, fair link transmissions, linear programming.

\section{INTRODUCTION}

Most Wireless Sensor Networks (WSN) application scenarios follow an energy-aware data gathering paradigm. The energy is usually managed by power control techniques which are widely applied in wireless networks $[4,18,17]$. However, we find that these techniques may often lead to cross-layer optimization problems. A typical example is the problem of Joint Link Scheduling and Power Assignment (JLSPA) which seeks to find an efficient link scheduling scheme, in which the power of sender nodes is set variable, such that certain requirements are satisfied.

In a time-driven WSN, sensor nodes need to send their data periodically. This period is usually known as a round of data-gathering. The time needed to perform a round will be determined by the schedule length which, on the other hand, is constrained by the interference effect. The interference is taken into account by considering the SINR parameter. Hence, the subject of this research is to find a feasible schedule such that concurrent link transmissions have fair quality under SINR constraint, or otherwise to solve the JLSPA problem.

The paper is organized as follows. In Section II we present a review of related works regarding the JLSPA problem. The power assignment problem for fair link transmissions is introduced in Section III. Here, we state in more details the motivation of this problem and propose a central approach solution. In Section IV, we propose an approach for the JLSPA problem, which allocates the links to time slots by applying the power assignment method discussed in the previous section. Finally, we conclude this work in Section V.

\section{RELATED WORK}

The JLSPA problem in WSNs has greatly attracted the attention of the networking community. By jointly solving these two basic problems, the network designers tend to satisfy the stringent requirements of WSN such as energy, PRR, and delay. As far as the scheduling problem apart is strongly concerned to interference avoidance for achieving successful multiple concurrent transmissions, the key point is the interference definition. Two basic definitions can be distinguished: the protocol and the physical one. The protocol definition of interference assumes that two links, which are less than $k$ hops $(k \geq 1, k \in N)$ away from each other, interfere potentially and cannot be scheduled in the same time slot. The indicated number of hops refers to the number of hops between the sender nodes of these links. On the other hand, the physical definition is based on the SINR constraint where the transmission links that do not satisfy the SINR constraint cannot be scheduled simultaneously ${ }^{1}$. However, as we will later see, the problem remains $\mathcal{N} \mathcal{P}$-hard regardless of the interference definition.

Following the protocol definition of the SINR, the scheduling problem needs to find a minimum-length schedule for all nodes in the network such that they will not

\footnotetext{
${ }^{1}$ In the following we will use interchangeably the terms transmission links and links
} 
interfere with each other. In the simple case $(k=1)$, the constraint requires that two edges in the same time slot do not share a node. The scheduling problem is widely modeled as the well-known optimization problem of graph coloring in which one seeks to find the minimal number of colors (chromatic number) necessary to color a graph such that no two adjacent nodes ${ }^{2}$ have the same color. Finding the chromatic number in a graph is $\mathcal{N} \mathcal{P}$-hard, therefore different methods have been proposed for this problem by the Operation Research community. These methods have been adapted and implemented for WSN, see for instance $[1,6,8,15]$.

As the protocol model underestimates the number of successful multiple concurrent transmissions, different works $[5,20,22]$ consider the cumulative interference proposed by the SINR model. For solving the link scheduling problem under physical interference, one approach seeks to classify the links according to their length (or distance). Hence, in $[10,20,21]$ each link belongs to a class $C_{k}$ if its length $l_{i}$ is between $2^{k} \leq\left|l_{i}\right|<2^{k+1}$. The idea behind the partition of links in classes is to schedule at the same time the links with the same length or very different one. The complexity of the proposed algorithm is $O\left(\log ^{4} n\right)$ where $n$ represents the number of nodes in the network. [16] identifies another condition that the set of links scheduled in the same time slot should satisfy. Given two links, this condition is related to the ratio between the distance of one link and the respective distance between the transmitter of this link and the receiver of the other one. The scheduling algorithm is a greedy one, which insures that the condition is satisfied if another link is added to the set of links scheduled in a given time slot. Goussevskaia et al. [10] design a greedy heuristic scheduling. For each class length $C_{k}$, they partition the area network into squares of length $a \cdot 2^{k}$ where $a$ is a constant and color the squares using 4 colors. Next, for each color they pick up the links having their receiver in different squares and assign them to a time slot. The process is repeated till all the links are scheduled. The same idea is revisited in [21]. In addition, they consider the case when the links have different demands to satisfy. Moreover, the length of the time slots is not fixed and the links may be scheduled more than once in a frame. In order to identify the links that can be scheduled simultaneously, in [21] is proposed a link classification based on the SNR (Signal to Noise Ratio) value. Instead of using the SINR threshold, Santi et al. [21] consider a graded SINR model which relates the PRR with SINR values as in Fig. 1. Their algorithm computes a schedule length of $O(r \cdot t)$, where $r$ is the maximal number of receivers in a cell, and $t$ is the time needed to transmit with the minimal SINR estimated in the frame ${ }^{3}$. For identifying the set

\footnotetext{
${ }^{2}$ Two nodes can be adjacent if they are ' $k$ ' hops away from each other.

${ }^{3}$ The time for transmitting a data unit is $t=\frac{1}{f}$ where $f$ is the data rate computed according to Shanon's channel capacity formula $f=B \cdot \log _{2}(1+S I N R)$ where $B$ is the channel bandwidth.
}

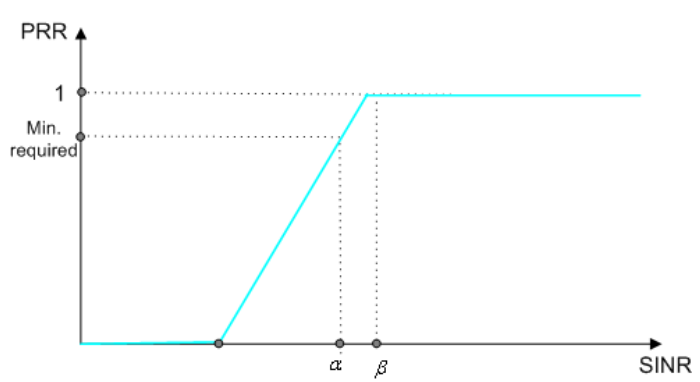

Figure 1: SINR graded model. $\alpha$ and $\beta$ are the lower and upper bounds of the desired SINR value, respectively, as described in Equations (2) and (4).

of concurrent links, another class of algorithms solves the maximum link matching problem ${ }^{4}$, see [13].

Despite the existence of different approaches for solving the scheduling problem under SINR constraint, designing a network protocol that takes in consideration this constraint is not trivial.

Till now, we have discussed the scheduling problem without emphasizing the power assignment strategy which is of paramount importance. Substantially, based on the above discussed problems we classify these strategies into three main groups:

Uniform power assignment. This is the simplest and the most intuitive case where it is assumed that all the sensors use the same power to transmit their data. Hence the question is to find the optimal transmission range that maximizes the number of multiple successful concurrent transmissions [11].

Linear power assignment. This scheme uses the rule of assigning the power proportionally to the signal attenuation ${ }^{5}$ which corresponds to the minimum power of transmission that guarantees a successful packet decoding from the receiver part. Similarly, the squareroot power assignments, proposed by [7] assigns the power proportionally with $\sqrt{d^{\gamma}}$. The linear and the uniform strategies are frequently used in MAC layer protocols

Nonlinear power assignment. According to this strategy the power will be disproportional to the link distance. The study of Moscibroda and Wattenhofer [20] shows that the uniform and linear power assignment may lead to inefficient scheduling as the shortest links may 'suffer' due to the high power signals emitted by the sources of the longest ones. Hence, in [20] is proposed a nonlinear power assignment strategy which gives priority to the short links. It assigns a minimal power to the longest links such that the communication is feasible and next, it increases with a scaling factor the power assigned to the shorter links.

\footnotetext{
${ }^{4}$ The maximum link matching seeks to find the maximum number of links in a given graph that do not have a node in common.

${ }^{5}$ The simplest model of signal attenuation is given by $d^{\gamma}$ where $d$ is the length of the link and $\gamma$ the path loss exponent.
} 
Using a different scaling factor, the same scheme is applied also in [16].

Related to the complexity of the problem of joint scheduling and power assignments, different variants of the problem are considered. Let refer at first to the link assignment problem. This problem needs to assign the links to different time slots such that two adjacent links will not be in the same time slot and the SINR constraints will be met for each of them. It is shown in [2] that this problem is at least as hard as the Edge Coloring Problem and is thus $\mathcal{N} \mathcal{P}$-hard. Further, the problem of determining a minimum length schedule that satisfies the SINR constraints is studied in [10]. By constructing a geometric instance of the scheduling problem, Goussevskaia et al. [10] show that the problem is reducible to the Partition Problem $^{6}$. The case when the schedule has to satisfy the links demands (or flow rates) is shown to be $\mathcal{N} \mathcal{P}$-hard by reducing it to the matching problem [3]. Hence, different variants of this problem and their respective complexities are discussed in the literature. The proof of the complexity of our JLSPA problem is presented in the work of Katz et al. [14]. The JLSPA problem was shown to be $\mathcal{N} \mathcal{P}$-hard by [14] if the network is embedded in the Euclidean plane and there are known upper and lower bounds on the power levels that can be used. Moreover, the proof remains true even for the case in which the sender node may choose its transmission power from an available set of discrete values.

\section{Power StRATEgy FOR FAIR LINK TRANSMissions}

\section{A. Research motivation}

As introduced in the previous section, the problem of joint link scheduling and power assignment may be basically solved by identifying the links that transmit simultaneously, and then by applying an efficient power control technique with the objective of minimizing the schedule length. This is also the main idea behind our general algorithm. In this section we will give in details the study on the power assignment problem under SINR constraint.

With respect to the power assignment problem, to the best of our knowledge, the previously proposed power assignment schemes fall into three categories: i) uniform ii) linear and iii) non linear ones (see Section II). In this study we will focus in designing an optimal power assignment strategy which assures fair link transmissions under SINR constraints.

For getting insights in modeling the power assignment problem, we were based in some experimental test provided in $[12,19,22]$. Considering only one transmitter node and one receiver node, and factoring out the issue of interference, it can be stated that the link quality between the transmitter and receiver improves as the transmitter

\footnotetext{
${ }^{6}$ Given a set of integers, the Partition problem seeks to decide whether it is possible to divide this set into two subsets, such that the sums of the numbers in each subset are equal.
}

increases its transmission power. This reasoning also holds for very sparse networks where the transmitter is only within the range of the intended recipient but not in the range of any other nodes that may be simultaneously receiving data from other transmitting nodes. Under these circumstances, existing transmission power control protocols, such as those found in PCBL [22], ATPC [19], ART [12], could help to ensure that an appropriate transmission power is used to achieve reliable link quality using minimum energy. These schemes generally use parameters such as the Received Signal Strength Indicator (RSSI) or Packet Receive Rate (PRR) to evaluate the quality of a link. This information is then used to take decisions about the transmission power that should be set to maximize link quality using the least amount of energy. However, as the network density increases and every transmitting node is potentially within range of multiple receivers, interference plays a much larger role. Under such circumstances parameters such as RSSI or PRR do not give a good indication of whether link quality is poor, or more importantly why it is poor. For example, if we assume that interference does not exist, a higher RSSI reading generally translates to a higher PRR (PCBL). However, as interference increases, a higher RSSI may not result in a higher PRR, as the increased RSSI may be due to other nodes that are transmitting simultaneously and are within the range of the receiver. In addition, some techniques as PCBL, ATPC and ART only depend on information available locally at a node to make deductions about the quality of a link. As it can be seen from the performance of ART, a node may not always be able to accurately differentiate between packet loss due to a weak signal or due to interference by using a localized approach. But as all nodes act independently of each other, one of drawbacks of such schemes is that nodes try to outdo each other. This results in higher power consumption and also has a detrimental effect on link quality. Due to these reasons, in this study we aim to design a centralized approach to perform transmission power control in a fair manner, such that every node that is actively transmitting in the network chooses a transmission power that minimizes the interference effects on all the non destination receivers. Our scheme aims to optimize the SINR parameter instead of only addressing RSSI or PRR as it is able to capture information about both the signal strength and interference more accurately. Finally, we model this problem as a maxmin linear programming one while having as an objective the maximization of the minimum value of SINR, and as constraint the RSSI and SINR values for each receiver.

\section{B. Notation and problem definition}

We model the wireless sensor network through a directed graph $G(V, E)$ where $V$ is the set of nodes representing the sensors, and $E$ is the set of links representing the wireless channel communication between the sensors. For each link $(i, j) \in E, i$ indicates the transmitter node 
and $j$ the receiver one. The weight of link $(i, j)$ is noted $\omega_{i j}$ and represents the attenuation of the signal. In some other context, $\omega_{i j}$ may be referred as gain if it would present the signal amplification to reach the receiver. We now assume that in a given time, only a subset of links $M$, where $M \subset E$ is activated. Let's assume that the incident nodes of the links $(i, j) \in M$ form the subset $T X$ and the others (the receiver nodes) the subset $R X$. Two properties can be noticed for $R X / T X$ subsets:

1) $R X \bigcap T X=\Phi$

2) $R X \bigcup T X \subseteq V$

The $S I N R_{j}$ value estimated in the receiver $j$, where $(i, j) \in M$ is given by the equation (1):

$$
S I N R_{j}=\frac{\frac{P_{i}}{\omega_{i j}}}{\sum_{k \in T X /\{i\}}\left(\frac{P_{k}}{\omega_{k j}}\right)+N_{a}}
$$

where $j \in R X, P_{i}$ and $P_{k}$ are the power assigned to the sender nodes, $\omega_{i j}$ notes the weight of the transmission link $(i, j), \frac{P_{k}}{\omega_{k j}}$ measures the interference of the other links over the receiver node of the link $(i, j)$, where $(i, j) \in M$, and $N_{a}$ is the floor noise which is considered as constant. Next, we define explicitly the parameters of a successful transmission. Clearly, a crucial parameter for estimating the link quality is PRR. This parameter is strongly related to SINR [21, 23]. According to these works, the packets are successfully received only when SINR exceeds a given threshold. The graded SINR model graphically presented in Fig. 1 shows the relation between PRR and SINR.

\section{Mathematical modeling}

In this section we present in detail the constraints and the objective function for this problem. First, in order to have fair link transmissions, we intend to maximize the minimum SINR value associated to receiver nodes. Moreover, this objective permits to improve the quality of the worst link which usually comes out to be a key point for measuring the network performance. Hence, we have a max-min problem. Let have a look at the constraints:

1) For having a successful transmission, we assume that the SINR value at the receiver has to be bigger than a threshold. We note $\alpha$ this lower bound as given in equation (2).

$$
S I N R_{j}=\frac{\frac{P_{i}}{\omega_{i j}}}{\sum_{k \in T X /\{i\}}\left(\frac{P_{k}}{\omega_{k j}}\right)+N_{a}} \geq \alpha
$$

2) The intended signal strength measured by Received Signal Strength Indicator (RSSI) indicator has to be bigger than a threshold $R S S I_{0}$. This threshold represents the lowest power level of the signal which permits a receiver to detect and decode the information of the signal. It is also known as receiver sensitivity and can be easily found in sensor data sheets.

$$
R S S I_{i j}=\frac{P_{i}}{\omega_{i j}} \geq R S S I_{0}
$$

where $R S S I_{i j}$ is the received strength indicator at the node $j$ when the link $(i, j)$ is activated, $(i, j) \in M$.

3) Considering the graded SINR model in Fig. 1, we can observe that beyond a given threshold $\beta$ of SINR, the PRR does not change. Imposing $\beta$ as an upper bound of SINR for all receivers could lead to scenarios solutions with a reduced number of nodes simultaneously transmitting. Nonetheless, the solution is less restricted if we ask:

$$
\min _{j \in R X} S I N R_{j} \leq \beta
$$

By itself, the replacement of $S I N R_{j} \leq \beta \forall j$ with (4) is not satisfactory for the problem (it is driven by the risk of infeasible solutions). But considering that we seek $\max \min S I N R$, we can claim to be closer to the initial constraint.

\section{Solution method}

The problem is modeled as a Mathematic Programming (MP). By summarizing the above constraints, we can write the following MP model:

$$
\begin{aligned}
& \operatorname{maximize} \min _{j \in R X} S I N R_{j} \\
& \text { s.t. : } \\
& S I N R_{j} \geq \alpha \quad \forall j \in R X \\
& \min _{j \in R X} S I N R_{j} \leq \beta \\
& \frac{P_{i}}{\omega_{i j}} \geq R S S I_{0} \quad \forall i \in T X,(i, j) \in M \\
& P_{\min } \leq P_{i} \leq P_{\max } \quad \forall i \in T X
\end{aligned}
$$

where the first and the second constraint guarantee that the minimum SINR value is between the lower $(\alpha)$ and the upper $(\beta)$ threshold. The third one guarantees that the signal in the receiver is sufficiently high for being detected and processed. And the forth emphasizes the fact that the node's power values should be in the interval $\left[P_{\min }, P_{\max }\right]$.

In the above MP formulation, we redefine the objective function. We note $z$ the minimum value of $S I N R_{j}$ and add the respective constraints. Finally, the objective and the constraints of the problem are given as follows: 
maximize $z$

s.t. :

$$
\begin{array}{ll}
\frac{\frac{P_{i}}{\omega_{i j}}}{\sum_{k \in T X /\{i\}}\left(\frac{P_{k}}{\omega_{k j}}\right)+N_{a}} \geq z \quad \forall(i, j) \in M \\
\alpha \leq z \leq \beta \\
\frac{P_{i}}{\omega_{i j}} \geq R S S I_{0} \\
P_{\text {min }} \leq P_{i} \leq P_{\text {max }} & \forall j \in R X,(i, j) \in M
\end{array}
$$

For this problem, the variables are given by the $P_{i}$ and $z$. We notice that the first constraint (equation 5) is a nonlinear inequality. Hence, our MP is nonlinear. To solve it, we begin by initially setting $z=\alpha$ in the first constraint. Because $\alpha$ is a lower bound for $z$, this assumption leads to a feasible solution (if such a solution exists for the initial problem). By assuming $z$ as a constant we obtain a LP model, called the problem $P 1$, which is formulated as follows:

$$
\text { maximize } \epsilon
$$

s.t. :

$$
\frac{P_{i}}{\omega_{i j}}-z \cdot\left(\sum_{k \in T X /\{i\}}\left(\frac{P_{k}}{\omega_{k j}}\right)+N_{a}\right)-\epsilon \geq 0 \quad(i, j) \in M
$$$$
\begin{aligned}
& \frac{P_{i}}{\omega_{i j}} \geq R S S I_{0} \quad \forall j \in R X,(i, j) \in M \\
& P_{\min } \leq P_{i} \leq P_{\max } \quad \forall i \in T X
\end{aligned}
$$

For sake of simplicity we use the same notation $z$ for both initial variable value and the current lower bound of $z$ which is updated (increased) constantly through iterations. In the constraint (9) (of the problem $P 1$ ), we have introduced the variable $\epsilon$ which will be helpful in increasing the $z$ value. Thus, $\epsilon$ is such that for each sender $i$ and receiver $j$

$$
\frac{\epsilon}{\sum_{k \in T X /\{i\}}\left(\frac{P_{k}}{\omega_{k j}}\right)+N_{a}}
$$

measures the gap between the $S I N R_{j}$ and $z$. Hence, by increasing $z$ according to (12) we ensure that there will be a feasible solution for the updated value of $z$.

$$
\min _{j \in R X} \frac{\epsilon}{\sum_{k \in T X /\{i\}}\left(\frac{P_{k}}{\omega_{k j}}\right)+N_{a}}
$$

Indeed, this is true since the last solution remains feasible for the updated $z$. The idea behind this is to gradually increase the $z$ value until we reach its maximum value. Algorithm 1 describes these operations.

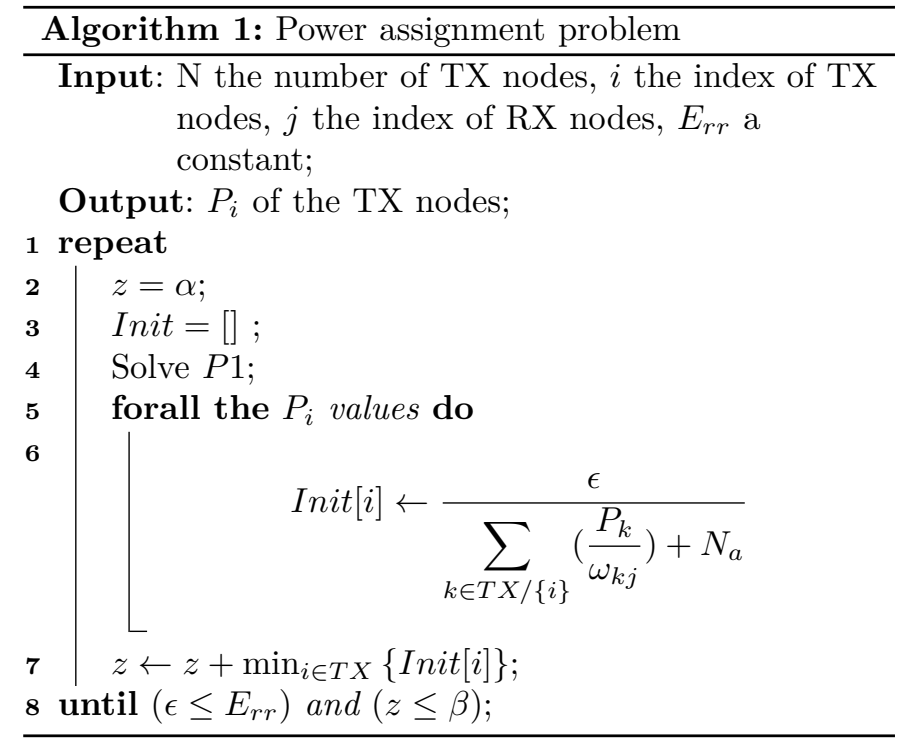

More precisely, the algorithm begins by solving the $P 1$ problem as defined above (with $z$ set to $\alpha$ ) and as result we obtain the $P_{i}$ values (or the power values assigned to sender nodes). The values stored in the Init vector, representing the ratio between $\epsilon$ and interference at each receiver (see formula (12)), are used to obtain the minimum value that allows to increase the SINR values while guarantying a feasible solution. The process is finite and the algorithm will stop when either $\epsilon$ becomes smaller than $E_{r r}$ or when the inferior bound of SINR becomes larger than $\beta$. In the latter case we set $z=\beta$, otherwise we take $z$.

\section{THE JLSPA PROBLEM}

In this section, we discuss the joint problem of link scheduling and power assignment strategy. For solving the JLSPA problem we need to perform the following tasks:

1) Identify the activated links.

2) Design a scheduling scheme.

3) Assign the power transmissions.

\section{A. Network topology and scheduling algorithm}

In order to identify the activated links that need to be scheduled, we assume that the sensors are uniformly deployed in a given area and the network is divided into clusters. Each cluster (or zone) has a cluster head which gathers the data from other sensors, aggregates and transmit them hop-by-hop to the Base Station (BS). The set of links connecting the cluster heads forms a data gathering tree rooted at BS and passing through all of them, as shown in Fig. 2. In order to find the optimal number of clusters and their respective distances, we refer to the network configuration algorithm proposed in [9]. We will take into consideration for scheduling only the links connecting the cluster heads. Hence, the topology provided by network configuration problem will serve as an instance 


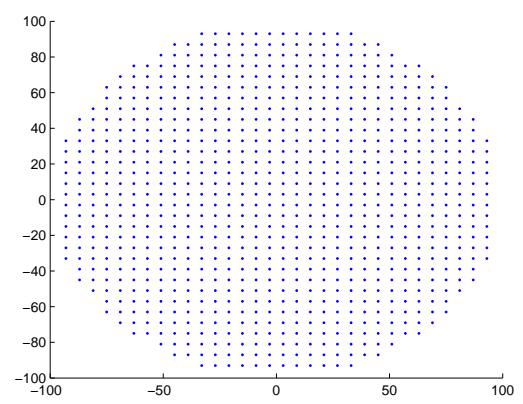

(a) A sensor field

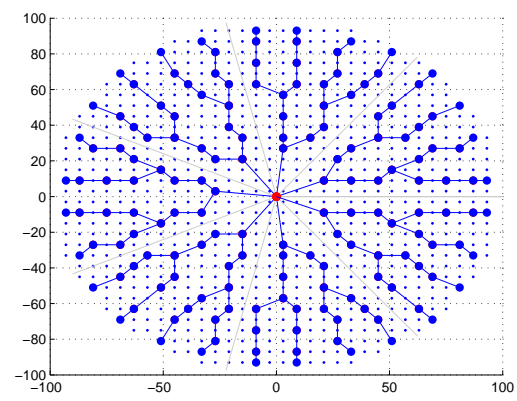

(b) A configured sensor network

Figure 2: Results of network configuration problem

to test the results of our proposed fair link transmission strategy ${ }^{7}$.

Regarding the scheduling, it can be modeled as the one-shot scheduling problem in [10], assuming that the links weight will be equal to one unit. As this problem is shown to be $\mathcal{N} \mathcal{P}$-hard, we propose a bottom-up approach described in Algorithm 2. This approach is a greedy heuristic which intends to put in a time slot the maximum number of links such that the SINR constraint is respected (where $S I N R_{\text {threshold }}$ represents the $\alpha$ value, see (2)). The set of links $L$ that need to be scheduled is given by solving the network configuration problem, as stated previously. In the set $L$ the links are sorted according to their respective coronas, meaning first we have the links of the corona closer to $B S$, next it will use the links of the second corona and so on. For each time slot, we try to put the links by beginning from those closer to the $B S$. The number of links that can be placed in a given time slot will be controlled by the three conditions given in lines $2-4$ of the test () function. The algorithm proceeds by taking into consideration each link that has not been assigned to a time slot. If the function test() returns True, the link in

\footnotetext{
${ }^{7}$ We remark that the scheduling problem, which takes into account the sensor node communications to the cluster head, can be easily solved by assuming that the respective communication links will be part of the activated set of links. Among the connected graph structures, we selected the tree as it presents one of the main used topologies for data gathering in WSN. However, we notice that our approach can be applied to arbitrary topologies of WSN.
}

consideration can be assigned to the current slot, otherwise the algorithm will check its validity for the next time slot.

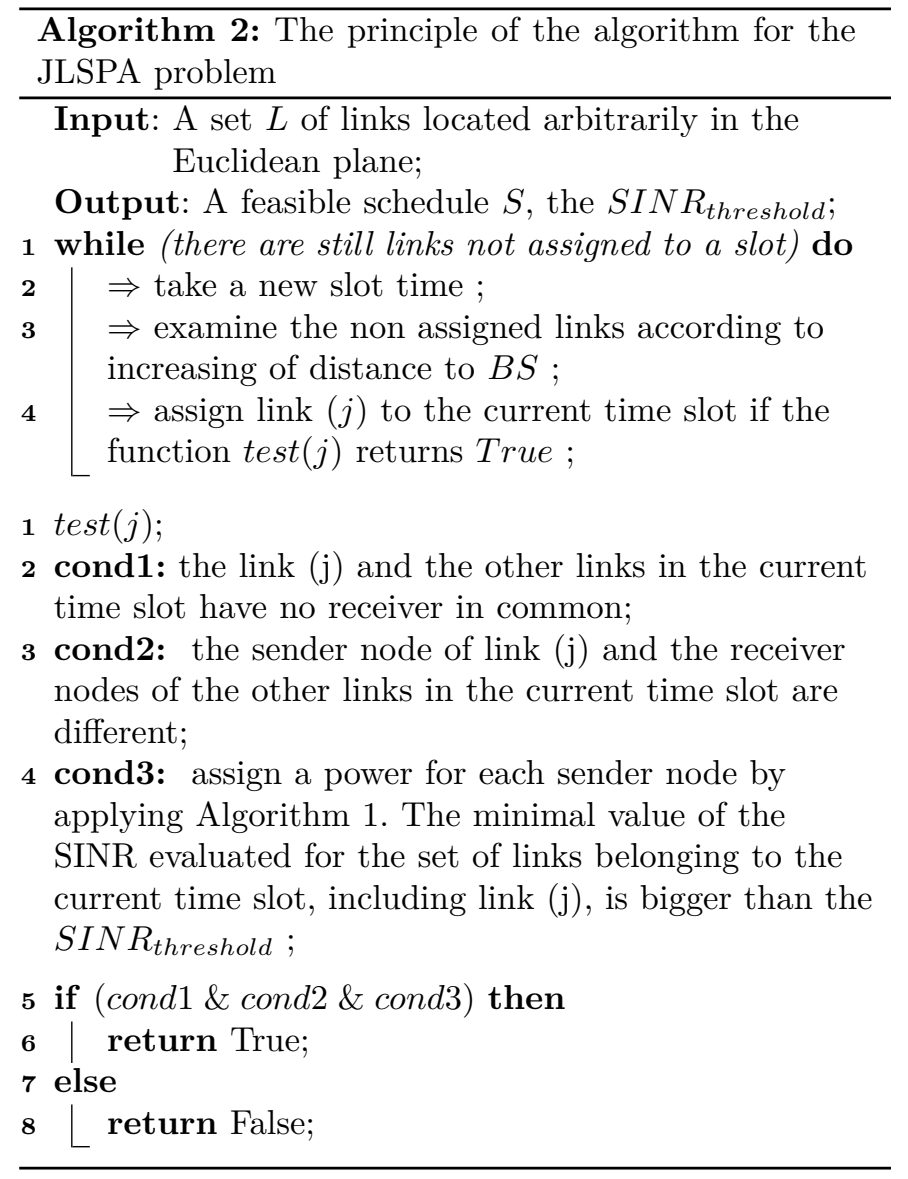

The heart of this algorithm is the SINR computation (see condition 3) which corresponds to the third task, the power assignment strategy. We propose Algorithm 1 to solve this problem. In this point one important question holds: How the power assignment strategy will effect the schedule length? The question is answered in the following section.

\section{B. Numerical results for the JLSPA problem}

In this section we will apply two different power assignment strategies i) the linear power assignment and ii) the power strategy for fair link transmissions (called fair SINR strategy below), to the scheduling algorithm proposed previously. The linear power assignment strategy consists of assigning a power to each activated link, which is proportional with the link weight.

To compute the link weights we use the log-distance path loss model. This model is formally expressed according to equation (13).

$$
\omega_{i j}\left(d_{i j}\right)=P L_{0}+10 \cdot \gamma \cdot \log _{10} \frac{d_{i j}}{d_{0}}+N(0, \sigma)
$$

where $d_{i j}$ is the transmitter $(i)$ - receiver $(j)$ distance, $d_{0}$ a reference distance, $P L_{0}$ the power decay corresponding to 
$d_{0}, \gamma$ the path loss exponent (rate at which signal decays), $N(0, \sigma)$ is a normal (or Gaussian) random variable with mean 0 and variance $\sigma$, reflecting the attenuation (in $\mathrm{dB}$ ) caused by flat fading. The value of weight $\omega_{i j}$ is given in $d B m$. The link weights are computed according to the model given in (13), but for computation simplicity we have not considered the $N(0, \sigma)$ value. For the rest of parameters, the reader can be referred to the table I.

Table I: Simulation parameters

\begin{tabular}{|c|c|c|c|}
\hline Type & II & Parameter & Value \\
\hline \multirow{6}{*}{ MP Parameters } & II & $\alpha$ & $1.99(3 \mathrm{~dB})$ \\
\hline & II & $R S S I_{0}$ & $-90 \mathrm{dBm}$ \\
\hline & II & $P_{\min }$ & $-25 \mathrm{dBm}$ \\
\hline & II & $P_{\max }$ & $0 \mathrm{dBm}$ \\
\hline & 11 & $\beta$ & $10 \mathrm{~dB}$ \\
\hline & 11 & $E_{r r}$ & $10^{-7}$ \\
\hline \multirow{3}{*}{ Channel Parameters } & II & $\gamma$ & 2 \\
\hline & II & Reference distance $d_{0}$ & $1 m$ \\
\hline & II & Power $P L_{0}$ & 52.4 \\
\hline \multirow{3}{*}{ Radio Parameters } & II & Noise floor & $-110 \mathrm{dBm}$ \\
\hline & II & White Gaussian noise $N_{w}$ & $4 \mathrm{~dB}$ \\
\hline & II & $N(0, \sigma)$ & 0 \\
\hline \multirow{3}{*}{ Network topology instance } & II & Network radius & $100 \mathrm{~m}$ \\
\hline & II & Inter nodes distance & $6 \mathrm{~m}$ \\
\hline & II & Number of activated links & 161 \\
\hline
\end{tabular}

The MP parameters in table I refers to the coefficients of MP presented in section III-D. For the $\alpha$ value we are referred to the Co-Channel Rejection Ratio (CCRR) defined in the sensor data sheets. From the empirical experiments provided in [24] we observed that the $\beta$ value can be approximated at $10 \mathrm{~dB}$. $R S S I_{0}, P_{\min }$ and $P_{\max }$ are extracted from the sensor $(\mathrm{MICAz})$ data sheet.

Our algorithm is coded in $\mathrm{C}++$ using the CPLEX 12.1 Library. The program is compiled with MSVC in a Windows environment, and all experiments were conducted on an AMD Opteron 2.60 GHz.

We have applied the linear and the fair power strategy to the scheduling problem and computed the schedule length for cases when the SINR threshold varies. These results are presented in Fig. 3. The fair power assignment strategy improves the schedule length with at least $31 \%$ (fair power assignment strategy requires 18 time slots with respect to linear schedule length which needs 26 time slots for a SINR threshold equal to 1.9 units). As we can observe, the number of slots required to schedule all the links has the tendency to increase for bigger values of SINR thresholds. Nonetheless, in particular cases this does not hold because the solutions of the greedy heuristic are not optimal.

Based on this result, we can conclude that the fair power assignment strategy can considerably reduce the schedule length. However, there is still place for further improving the scheduling heuristic.

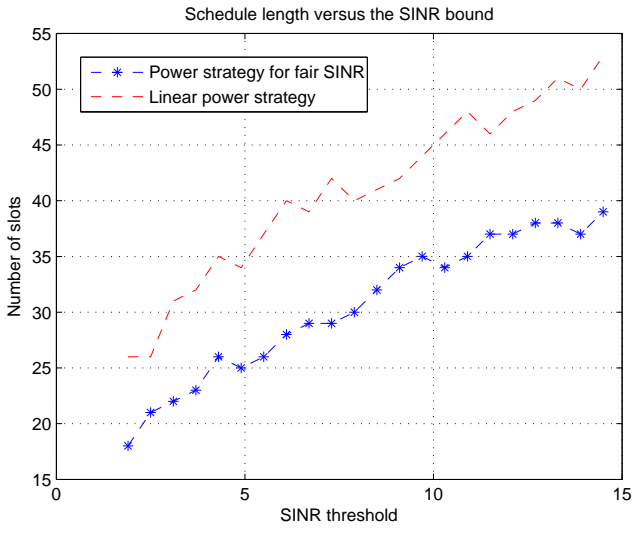

Figure 3: Time slots number versus SINR

\section{Future WORK}

The JLSPA problem is one of the main problems in the WSN design. It consists in finding a feasible schedule and a power assignment scheme such that the schedule length is minimized and the concurrent transmissions have a fair quality in terms of SINR. The decisions of this problem impact different layer parameters and more precisely: the power in the physical layer, the wake-up time managed by the MAC layer, the PRR (estimated in relation with SINR) belonging to application layer. To solve the problem we proposed a greedy heuristic which intend to put in a time slot the maximum number of links such that the SINR constraint is respected. Whereas for the power assignment strategy, we seek to assign to each node such a power that the minimal SINR value estimated at each receiver is maximized. This problem is solved optimally and to the best of our knowledge, we are the first to propose an exact method. The SINR parameter is strongly related to the PRR. Hence, by respecting the SINR threshold at each receiver, and assuring a fair SINR, we can realize a fair link quality (in terms of PRR) for WSN.

Different problems may be interesting to be investigated as future work, such as:

- Improve the scheduling algorithm in order to have lower bounds for the JLSPA problem.

- Develop an adaptive transmission power control algorithm that operates in a distributed manner. The algorithm should be able to adapt its transmission power quickly to suit a rapidly changing radio environment.

\section{REFERENCES}

[1] T.A. Al-Khdour and U. Baroudi. An energy-efficient distributed schedule-based communication protocol for periodic wireless sensor networks. Arabian journal for science and engineering, 35:155-168, 2010.

[2] P. Björklund, P. Värbrand, and D. Yuan. Resource optimization of spatial TDMA in ad hoc radio net- 
works: A column generation approach. INFOCOM, 2:818-824, 2003.

[3] S.A. Borbash and A. Ephremides. Wireless link scheduling with power control and SINR constraints. IEEE Trans. on Inf. Theory, 52(11):5106 - 5111, 2006.

[4] M. Cardei and I. Cardei. Energy-efficient range assignment in heterogeneous wireless sensor networks. Wir. and Mob. Com. ICWMC' '06., pages 11-22, 2006.

[5] D. Chafekar, V.A. Kumart, M.V. Marathe, S. Parthasarathy, and A. Srinivasan. Approximation algorithms for computing capacity of wireless networks with SINR constraints. INFOCOM, pages 1166 - 1174, 2008.

[6] S. Ergen and P. Varaiya. TDMA scheduling algorithms for WSN. Wireless Networks, 16:985 - 997, 2010.

[7] A. Fanghänel, T. Kesselheim H. Räcke, and B. Vöcking. Oblivious interference scheduling. Proc. of the ACM PODC, pages 220-229, 2009.

[8] S. Gandham, M. Dawande, and R. Prakash. Link scheduling in sensor networks: distributed edge coloring revisited. INFOCOM, 4:2492-2501, 2005.

[9] A. Gogu, D. Nace, and Y. Challal. Energy-aware network configuration for wireless sensor networks. Network-Based Information Systems (NBiS), pages 22-29, 2011.

[10] O. Goussevskaia, Y.A. Oswald, and R.Wattenhofer. Complexity in geometric sinr. MobiHoc, pages 100109, 2007.

[11] P. Gupta and P. R. Kumar. Critical power for asymptotic connectivity in wireless networks. Stochastic Analysis, Control, Optimization and Applications: Book, pages 547-566, 1998.

[12] G. Hackmann, O. Chipara, and C. Lu. Robust topology control for indoor wireless sensor networks. SenSys, pages 57-70, 2008.

[13] Q.S. Hua. Scheduling wireless links with SINR constraints. PhD thesis, The university of Hong Kong, 2009.

[14] B. Katz, M. V olker, and D. Wagner. Energy efficient scheduling with power control for wireless networks. Modeling and Optimization in Mobile, Ad Hoc and Wireless Networks (WiOpt), pages 160-169, 2010.

[15] R. Kawano and T. Miyazaki. Distributed data aggregation in multi-sink sensor networks using a graph coloring algorithm. In Proceedings of IEEE AINA, pages 906-912, 2009.

[16] T. Kesselheim. Constant-factor approximation for wireless capacity maximization with power control in the sinr model. Symp. on Discrete Algorithms (SODA), pages 1549-1559, 2011.

[17] D. Li, H. Du, L. Liu, and S-C. Huang. Joint topology control and power conservation for wireless sensor networks using transmit power adjustment. Proc. of the 14th Conf. on Comp. and Combinatorics, pages 541-550, 2008.
[18] M. Li and B. Yang. A survey on topology issues in wireless sensor network. In Proc. of the Int. Conf. on Wireless Networks, page 503, 2006.

[19] S. Lin, J. Zhang, L. Gu, T. He, and J. Stankovic. Atpc: Adaptive transmission power control for wireless sensor networks. SenSys, pages 223-236, 2006.

[20] T. Moscibroda and R. Wattenhofer. The complexity of connectivity in wireless networks. INFOCOM, pages 1-13, 2006.

[21] P. Santi, R. Maheshwari, G. Resta, S. Das, and D. M. Blough. Wireless link scheduling under a graded sinr interference model. FOWANC, pages 3-12, 2009.

[22] D. Son, B. Krishnamachari, and J. Heidemann. Experimental study of the effects of transmission power control and blacklisting in wireless sensor networks. SECON, pages 289-298, 2004.

[23] A. Sridharan and B. Krishnamachari. Max-min fair collision-free scheduling for wireless sensor networks. Perfor., Comp. and Communic., 585-590, 2004.

[24] M. Z. Zamalloa and B. Krishnamachari. An analysis of unreliability and asymmetry in low-power wireless links. ACM Trans. Sen. Netw., 3, June 2007. ISSN 1550-4859. 